\title{
A Study on the Mobile Media Transmission Mode and the Corresponding Marketing Strategies
}

\author{
Zhifang Qian \\ Yiwu Industrial\& Commercial College, Yiwu City, Zhejiang Province, \\ 322000, China,
}

\begin{abstract}
With the development of China's mobile communication technology and the popularity of $4 \mathrm{G}$ network, mobile phone begins to shift from a single communication tool to the mass media, and its media functions and spread advantages continue to appear. It has become the product of the combination of communication technology and computer technology. Based on the author's learning and practical experience, this paper first explained the characteristics of mobile phone as a marketing media, and then analyzed the spread model of mobile media, and finally put forward the mobile media business model. Fully grasping the development situation of mobile media and the latest trends has a positive significance for marketing personnel to plan marketing strategies and to explore new marketing model.
\end{abstract}

Keywords: Mobile media; Marketing Characteristics; Propagation Model; Marketing Strategy

\section{Introduction}

Mobile Internet and touch media provide a good foundation for the development of we-media and the innovation of traditional propagation model. This provides new opportunities for the development of enterprises and also changes the way people pick up information. The use of the mobile internet makes the information gradually universal, and anyone can publish and receive information in the mobile network platform. Moreover, people have a strong voice in the process of 
using mobile media, and everyone can become a seller. Companies speak for their own products and become media people. In order to seek the development, modern enterprises constantly look for new ways of marketing communication[1] . In the traditional communication environment, advertisement is the most important marketing model. Modern enterprises are good at seizing the development features of present new media to update their ideas, using the rapidity and participation of mobile media information dissemination to spread their brand culture, effectively using media platform to integrate information and innovating marketing mode to promote the development of enterprises.

\section{The Features of Mobile Phone Acting as a Marketing Media}

\subsection{Cover a Wide Range of People}

According to the Thirty-Seventh China Internet Development Statistics Report released by China Internet Network Information Center (CNNIC), China's Internet users had reached 688 million up to December 2015, and there were 39 million 510 thousand new Internet users for the entire year. The internet penetration rate was $50.3 \%$, and it increased by 2.4 percentage points compared with the end rate of 2014. The number of China's mobile phone users reached 620 million, and the proportion of mobile Internet users increased from $85.8 \%$ in 2014 to $90.1 \%$. It follows that mobile phone not only has a large number of users, but also owns a relatively stable user number because its communication service is viscous, and with the expansion of mobile phone function, the number of mobile phone users will increase[2]. Therefore, using mobile phone as a marketing media and having a huge marketing object as the basis have become an important feature of mobile phone marketing.

\subsection{High Viscosity}

With the continuous expansion of the function, the mobile phone has transferred from the simple voice tool to the media of information dissemination. The dainty and portability of mobile phone make it easy to be carried and to ensure personal privacy, so the high frequency of use and contact become inevitable, so that the mobile phone is highly viscous. The information spread through mobile phone is also more targeted, time-sensitive, and permeable, making marketing communications not be limited by time and place.

\subsection{Interactivity}

The use of wireless network on the mobile phone is convenient for people to communicate and interact in virtual platforms, such as web forum, chat software, micro-blog blog and so on, therefore, people can enjoy all kinds of virtual communication in the Internet just like in the computer. Moreover, the mobile phone has broken through the inconvenience of the computer, and becomes an 
important platform for users to interact with virtual network. Taking the mobile phone as the carrier, the development of a variety of instant communication software, such as WeChat, Ali Wangwang, Micro Shop and Pat, have become an important medium for the interaction between enterprises or businesses and consumers. Through these mobile phone intelligent software, users not only are the recipients of the information, but also become the active information publisher and information requester. This interactive relationship is not only conducive to enterprises conveying information according to the actual situation but also can make enterprises get users' timely feedback to improve product quality and service level and enrich the means of marketing.

\section{The Propagation Mode of Mobile Media}

In the propagation process of mobile media, it breaks the original mass media spread. The mobile media not only has access to the mass media information, but also has strong interpersonal communication characteristics. Also, it shows the multiple interaction between the communicator and the receiver, and embodies the characteristics of the decomposition and collection of the rights in the new propagation mode, thus effectively breaking through the institutional nature and the authority of traditional mass media[3].

\subsection{The Interpersonal Propagation Mode of Peer to Peer}

In the interpersonal communication model of peer to peer, the mobile phone users have dual identities, and they are the sender and reducer of the information symbol. Mobile phones not only provide users contents or popular information sent by service providers but also establish a network of interpersonal communication between user groups, thus the mass information can also be transmitted through the interpersonal communication channel and the interaction between the individual and the individual is interactive. In addition, there is no third party between the information receiver and sender, the process of sending and receiving information is very fast and both sides are equal. The communication mode of point to point has become an important feature of mobile phone media.

\subsection{The Transmission Mode of One to Many}

Mobile media can transfer various single way of communication model into complex and integrated modes of transmission on the new media communication platform. In the mobile media communication model of one to many, SP, I-mode, WAP and 4G site become communicators, and they can collect, process, filter and send information[4]. These communicators not only have the personal characteristics of the communication, at the same time, mobile phone users will send some of their useful information to other users. The convenient forwarding function of mobile phones let the utility of influence center exceed the time limits. 


\section{Mobile Media Business Model}

Mobile media has the advantages of strong interaction and low costs, which can provide the unique interactive function. Cooperation can enhance the traditional media audience participation, and then gets more market spaces. The traditional media have many advantages, for example, high market shares, mature business model and a fixed loyal audience. Therefore, for the mobile media industry, the complementary with the traditional media can achieve a win-win profit.

\subsection{The Model of Fusion with the Newspaper Media}

Newspaper media is flexible to management and has a high market coverage rate, also its contents are relatively mature, but the transfer rate is low and it is hard to preserve. However, the mobile phone media makes up for the the newspaper media's low transmission rate. The integration of the two sides complement each other's shortcomings, and fully play their respective advantages. The production of mobile phone newspapers and mobile phone novels makes the mobile phones interact with newspapers. With the help of telecom operators, users can read and receive books at any time. The mobile phone newspaper is illustrated, and it contains all the information in the newspaper, it can also support the interaction between the user and the newspaper. The cross platform collaboration between traditional media and mobile media gives birth to a new model of news dissemination[6].

\subsection{The Model of Fusion with the Broadcast Media}

The characteristics of mobile media can make up for the shortcomings of poor interaction and information storage of broadcast media. And with the enhance of the integration of the mobile phone terminal function, such as the installation of radio software and built-in FM receiver module and calling a specific service call make the phone has a radio function. The user listens to the public and economical radio program on the radio, and downloads some of the charges on the phone, along with his favorite radio programs or music. The convergence of mobile media and broadcast media realizes the fuse of the popularity and diversity. In the respective advantages of the two media, the convenience of mobile media is more prominent.

\subsection{The Model of Fusion with the TV Media}

The convergence of mobile media and television media makes programs on TV broadcast through the mobile media. This model makes the advantages of both mobile phones and televisions be focused on the phone. Phone TV includes digital broadcasting and mobile network transmission mode, which makes the mobile phone industry chain link media have generated great interests. Coupled with the recent definition of mobile phone TV standards, mobile phone TV will become an important form of mobile phone media. 


\subsection{The Model of Fusion with the Internet Media}

All aspects of the mobile media industry chain have begun to promote the integration of mobile media and the Internet. In particular, terminal manufacturers develop more products to support these businesses, such as mobile music, animation, games and business transactions and so on. At the same time, in order to promote the common development of mobile phone media and the Internet, mobile operators and Internet sites also carry out all kinds of cooperation which is conducive to the integration of mobile media and Internet, including content sharing and network convergence[7].

\subsection{The Model of Fusion with the Advertising Industry}

The advantage of mobile advertising is the high rate of information transmission. Advertisements have a strong pertinence. Businesses publish advertisements through the mobile phone media, so that customers can understand the information which they need in a timely manner, operators also get a huge amount of information, and it improves the utilization of the network. The current forms of mobile phone media advertising include text, image, video and other ways. And according to the creative demands, businesses can create various, lively and attractive advertisements. With the development of mobile media, advertising revenue will become the most important source of profit for mobile media. Mobile media advertising can takes the form of establishing the home page and selling pages according to the categories, but also can take the form of implantable, broadcast, on-demand, etc.

\section{Conclusion}

Mobile phones can not only be used to publish news, read books and newspapers, watch online etc., but also can be used for video dialogue, music downloads, chat shopping, etc. Mobile media, which is based on the mobile phone, appears after the emergence of newspaper, radio, television and network. It not only has the characteristics of mobile communication's mobility, individuality, real-time, safety, but also has unique advantages of wider popularity, stronger interaction, more convenient portability compared to other four media. The integration of the rapid development of mobile communication technology and the development of commercial society make the mode of transmission and operation of mobile phone media becomes more specific and vivid, and mobile phone has changed from a simple voice communication tool into a new marketing media. In the times of new media broadcast, how to use phones' advantages to promote the integration of mobile phone and mobile phone media marketing and other media and to enhance the efficiency of their own media, and to explore a more suitable way of marketing, are common pursuits of mobile operators and suppliers. We believe that with the continuous norms of the industry, the development of mobile media and business model will be more beautiful. 


\section{References}

[1] Li Wanting. Mobile media profit model and marketing strategy analysis. news world, 03, 74-75, 2012.

[2] Chunlin, Lu you. Mobile phone media marketing model of. news enthusiasts, 23, pp.27-28, 2009.

[3] Chunlin, Lu strong marketing research. Mobile phone media writing and editing, 06, pp.61-62, 2009.

[4] Du Xiaonian. Analysis of the communication mode of the mobile phone. precision marketing modern economic information, 0, pp.300-301, 2014.

[5] Curtis Zixia. Social media marketing communication mode. press 24, 62-67, 2014 ,.

[6] Zhou Yang. Mobile phone media marketing mode research on. News Herald, 10. pp. $280,2015$.

[7] Li. A new era of mobile media marketing communication model. Drama House, 12, pp.241, 2015. 